\title{
The final phase of inspiral of neutron stars: realistic equations of state
}

\author{
D. Gondek-Rosińska ${ }^{\mathrm{a}, \mathrm{b}, \mathrm{c}, \mathrm{d}}$, M. Bejger ${ }^{\mathrm{d}}$, T. Bulik ${ }^{\mathrm{e}, \mathrm{d}}$, \\ E. Gourgoulhon ${ }^{c}$, Paweł Haensel ${ }^{\mathrm{d}, \mathrm{c}}$, F. Limousin ${ }^{\mathrm{c}}$ \\ K. Taniguchi ${ }^{\mathrm{f}}$ L. Zdunik ${ }^{\mathrm{d}}$ \\ a Departament de Fisica Aplicada, Universitat d'Alacant, Apartat de correus 99, \\ 03080 Alacant, Spain \\ ${ }^{\mathrm{b}}$ Institute of Astronomy, University of Zielona Góra, Lubuska 2, 65-265, Zielona \\ Góra, Poland \\ ${ }^{\mathrm{c}}$ LUTH, Observatoire de Paris, Universite Paris 7, Place Jules Janssen, 92195 \\ Meudon Cedex, France \\ ${ }^{\mathrm{d}}$ CAMK, Bartycka 18, 00716, Warsaw, Poland

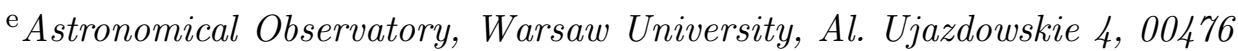 \\ Warsaw, Poland \\ ${ }^{\mathrm{f}}$ Department of Physics, University of Illinois at Urbana-Champaign, Urbana, \\ Illinois 61801, USA
}

\begin{abstract}
Coalescing compact star binaries are expected to be among the strongest sources of gravitational radiation to be seen by laser interferometers. We present calculations of the final phase of inspiral of equal mass irrotational neutron star binaries and strange quark star binaries. Six types of equations of state at zero temperature are used - three realistic nuclear equations of state of various softness and three different MIT bag models of strange quark matter. We study the precoalescing stage within the Isenberg-Wilson-Mathews approximation of general relativity using a multidomain spectral method. The gravitational-radiation driven evolution of the binary system is approximated by a sequence of quasi-equilibrium configurations at fixed baryon number and decreasing separation. We find that the innermost stable circular orbit (ISCO) is given by an orbital instability for binary strange quark stars and by the mass-shedding limit for neutron star binaries. The gravitational wave frequency at the ISCO, which marks the end of the inspiral phase, is found to be $\sim 1100-1460 \mathrm{~Hz}$ for two $1.35 M_{\odot}$ irrotational strange stars described by the MIT bag model and between $800 \mathrm{~Hz}$ and $1230 \mathrm{~Hz}$ for neutron stars.
\end{abstract}

Key words:

gravitational waves-relativity- stars:binaries- stars: neutron- equation of state

Preprint submitted to Elsevier Science 15 August 2018 


\section{Introduction}

Coalescing neutron star binaries are considered among the strongest and most likely sources of gravitational waves to be seen by VIRGO/LIGO interferometers [11, 23, 5]. Due to the emission of gravitational radiation, binary compact stars decrease their orbital separation and finally merge. We separate the evolution of a binary system into three phases : point-like inspiral where orbital separation is much larger than the neutron star radius, hydrostationary inspiral where orbital separation is just a few times larger than the radius of the neutron star so that hydrodynamics play an important role, and merger in which the two stars coalesce dynamically. Gravitational waves emitted during last orbits of inspiral phase and the merger phase could yield important information about the equation of state (EOS) of dense matter [14, 32, 29, 4, 25]. Up to now, all relativistic calculations (except those of [29, 4, 25]) of the last orbits of inspiral phase have been done for the simplified EOS of dense matter the polytropic EOS. In the paper we present results of our studies on late stage of inspiral of binary systems containing equal mass compact stars described by different realistic EOS of dense matter. The calculations are performed in the framework of Isenberg-Wilson-Mathews approximation to general relativity (see Ref. [3] for a review). We consider binary systems consisting of two identical stars. We choose the gravitational mass of each star to be $1.35 M_{\odot}$ at infinite separation in order to be consistent with recent population synthesis calculations [9, 19] and with the current set of well-measured neutron star masses in relativistic binary radio pulsars [26, 10]. We assume that the velocity flow in the stellar interiors is irrotational (the fluid has zero vorticity in the inertial frame) since the viscosity of neutron star matter (or strange

star matter) is far too low to ensure synchronization during the late stage of the inspiral [6, 24].

\section{Equations of state and stellar models}

In Fig. 1 we show gravitational mass versus stellar radius for sequences of static compact stars. We limit ourselves to neutron stars consisting of nucleons and hyperons and strange quark stars described by the MIT bag model. Depending on the EOS we obtain the radius of a $1.35 M_{\odot}$ star in the range $10-14 \mathrm{~km}$. We perform calculations for three nuclear EOS of dense matter based on modern

Email address: Dorota.Gondek@obspm.fr (D. Gondek-Rosińska). 


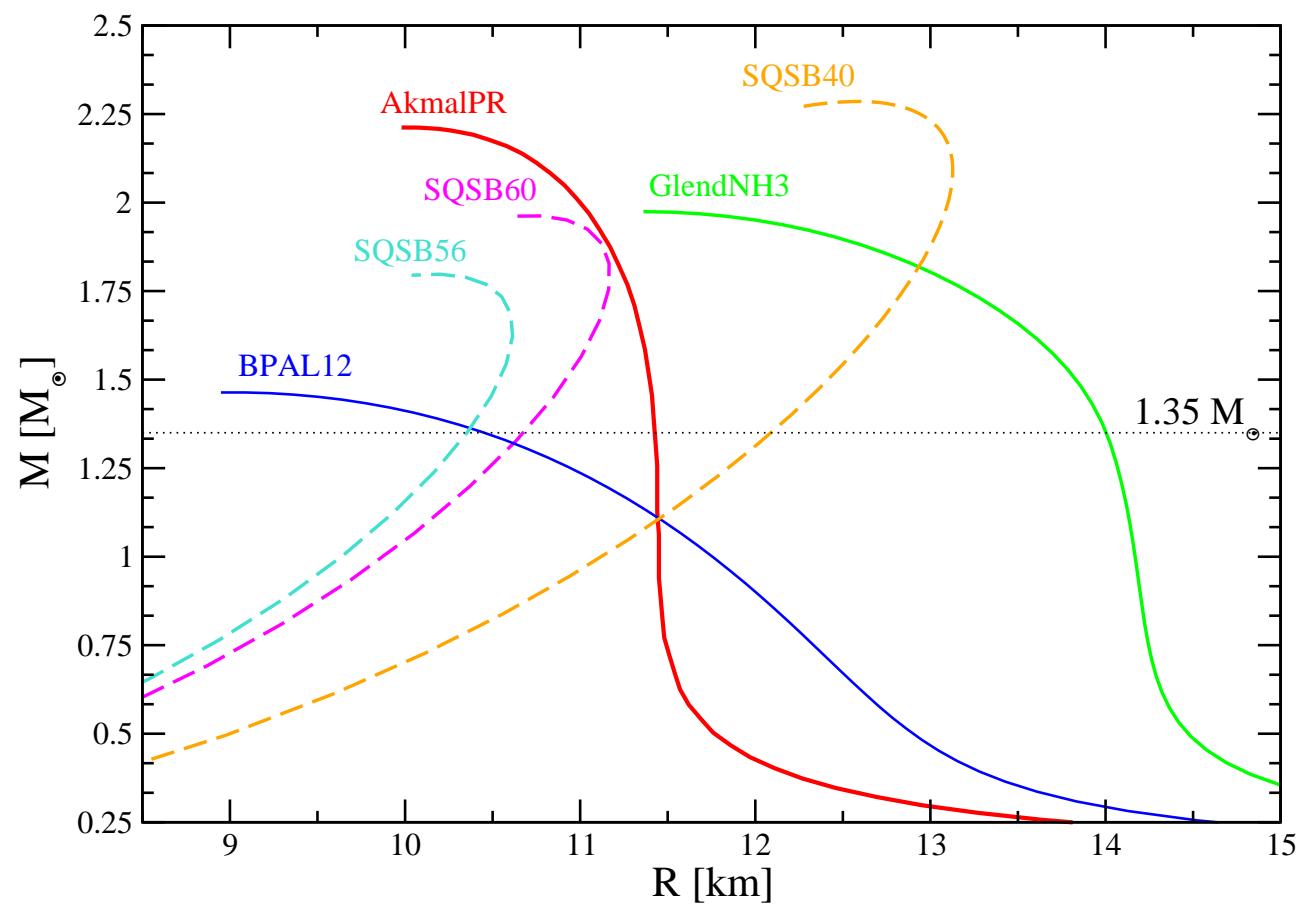

Fig. 1. Gravitational mass $M$ versus stellar radius $R$ for sequences of static neutron stars described by three different nuclear equations of state (solid lines) and strange quark stars described by different MIT bag models (dashed lines).

many-body calculations. The differences in the M-R relation for stellar models of neutron stars (solid lines) shown in Fig. 1 reflect the uncertainties in the existing theories of the interactions in nuclear matter. We consider one soft (BPAL12, [8]) and one stiff (AkmalPR, [2]) EOS of matter composed of nucleons, electrons and muons. We considered also one EOS in which hyperons are present at high densities (GlendNH3, [20]). The neutron star crust is described by means of a realistic EOS obtained in the many-body calculations (see [4] for details). We note that the equations of state of nuclear matter can be considered static, as the timescales for bulk processes such as viscosity is much longer than the last stages of inspiral considered here.

Strange stars are currently considered as a possible alternative to neutron stars as compact objects (see e.g. [33, 27, 18] and references therein). Typically, they are modeled with an EOS based on the MIT-bag model in which quark confinement is described by an energy term proportional to the volume (e.g. [1, 21]) in which quark confinement is described by an energy term proportional to the volume [15]. There are three physical quantities entering the MIT-bag model: the mass of the strange quarks, $m_{\mathrm{s}}$, the bag constant, $B$, and the strength of the QCD coupling constant $\alpha$. In the framework of this model the quark matter is composed of massless $\mathrm{u}$, $\mathrm{d}$ quarks, massive s quarks and electrons. Strange stars are self-bound objects, having high density (in the range $\left.\sim 3-6.4\left[10^{14} \mathrm{~g} / \mathrm{cm}^{3}\right][18]\right)$ at the surface. Dashed lines in Fig. 1 correspond to sequences of static strange quark stars described by three dif- 


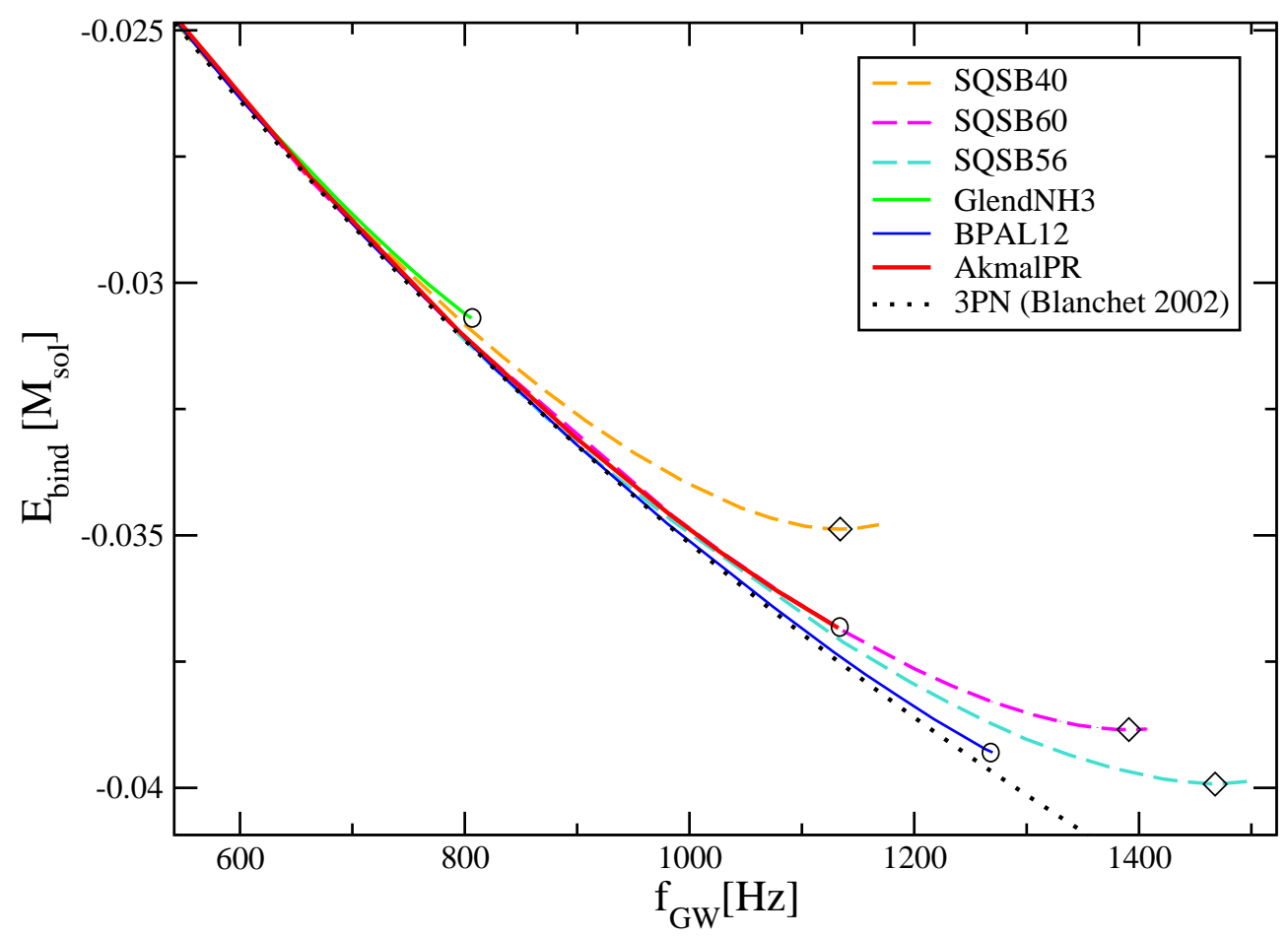

Fig. 2. Binding energy as a function of gravitational wave frequency (twice the orbital frequency) along evolutionary sequences of irrotational binaries. The solid lines denote neutron stars, the dashed one strange quark stars and the dotted line point-mass binaries in the third order Post-Newtonian approximation [7]. Each evolutionary sequence finishes at the innermost stable circular orbit. The diamonds correspond to dynamical orbital instability while circles to the mass-shedding limit.

ferent sets of parameters of the MIT-bag model: SQS56 - the standard MIT bag model: $m_{\mathrm{s}} c^{2}=200 \mathrm{MeV}, \alpha=0.2, B=56 \mathrm{MeV} / \mathrm{fm}^{3}$; SQSB60 - the simplified MIT bag model with $m_{\mathrm{s}}=0, \alpha=0 ; B=60 \mathrm{MeV} / \mathrm{fm}^{3}$; SQSB40 the "extreme" MIT bag model (relatively low strange quark mass and $B$ but high $\alpha$ ): $m_{\mathrm{s}} c^{2}=100 \mathrm{MeV}, \alpha=0.6, B=40 \mathrm{MeV} / \mathrm{fm}^{3}$.

\section{Numerical method}

In the inspiral phase, since the timescale of orbital shrinking due to the emission of gravitational waves is longer than the orbital period, one may consider a binary system to be in a quasi-equilibrium state (helical Killing vector approximation). For each EOS, we construct so called an evolutionary sequence by calculating a sequence of quasi-equilibrium configurations with fixed baryon mass and decreasing orbital separation. We assume that the timescale in which the neutron star adjusts to the current gravitational potential is much shorter that the timescale of the orbital shrinking. The present computations of close binary neutron star or strange star systems rely on the assumption of irro- 
tational flow of the fluid and a conformally flat spatial 3-metric (IsenbergWilson-Mathews approximation). In order to calculate the last orbits of inspiral phase of binary compact stars we use a numerical code which solves the five coupled, nonlinear, elliptic equations for the gravitational field, supplemented by an elliptic equation for the velocity potential of irrotational flows (see 25] for a discussion on different boundary conditions in the case of strange stars and neutron stars). The code has been already used successfully for calculating the final phase of the inspiral of compact stars [30, 31, 32, 4, 25]. This code is built upon the $\mathrm{C}++$ library LoRENE (http://www.lorene.obspm.fr). The complete description of the resulting general relativistic equations, the whole algorithm, as well numerous tests of the code can be found in [17]. Additional tests have been presented in Sect. III of [32].

\section{Results}

In Fig. 2 we show the evolution of equal mass binary neutron stars (solid lines) and strange stars (dashed lines) having total gravitational mass $2.7 M_{\odot}$ at infinity. The binding energy $E_{\text {bind }}$ is defined as the difference between $M_{A D M}$ (Arnowitt-Deser-Misner mass - the total mass-energy of a binary system [32]) and the total mass of the system at infinite separation. This energy is equal to total energy emitted by a binary system in gravitational waves. The frequency of gravitational waves is twice the orbital frequency. Comparison of our numerical results with 3rd order PN point masses calculations [7] reveals a good agreement for small frequencies (large separations). The deviation from PN curves at higher frequencies (smaller separation) is due to hydrodynamical effects, which are not taken into account in the PN approach. A turning point of $E_{\text {bind }}$ along an irrotational evolutionary sequence indicates the orbital (dynamical) instability [16]. This instability originates both from relativistic effects and hydrodynamical effects. In the case where no turning point of $E_{\text {bind }}$ occurs along the sequence, the mass-shedding limit (Roche lobe overflow) marks the end of the inspiral phase of the binary system, since recent dynamical calculations for $\gamma=2$ polytrope have shown that the time to coalescence was shorter than one orbital period for configurations at the mass-shedding limit (i.e. see [28]). Thus the physical inspiral of binary compact stars terminates by either the orbital instability (turning point of $E_{\text {bind }}$ ) or the mass-shedding limit. In both cases, this defines the innermost stable circular orbit (ISCO). The end of inspiral phase strongly depends on EOS - for irrotational neutron star binaries a quasi-equilibrium sequence terminates by mass-shedding limit (circles at the end of each line in Fig 2) and for strange stars by orbital instability (shown as diamonds in Fig. 2). The differences in the evolution of binary strange stars and neutron stars stem from the fact that strange stars are principally bound by additional force than gravitation: the strong interaction between quarks. 
They are self-bound objects having very high adiabatic index at the stellar surface (see [25]). Although the crust of a $1.35 M_{\odot}$ neutron star contains only a few percent of the stellar mass, this region is easily deformed under the action of the tidal forces resulting from the gravitational field produced by the companion star. The end of inspiral phase of binary stars strongly depends on the stiffness of matter in this region. The frequency of gravitational waves at the ISCO is one of potentially observable parameters by the gravitational wave detectors. In addition so called "break frequency", a characteristic frequency where the power emitted in gravitational waves decreases measurably [14, 22], could be an observable quantity. The frequencies of gravitational waves at the depature point from the 3PN aproximation and at the ISCO strongly depend on the EOS. For irrotational equal mass (of $1.35 M_{\odot}$ at infinite separation) binaries the ISCO frequency is $\sim 1100-1460 \mathrm{~Hz}$ for strange stars described by the MIT bag model and between $800 \mathrm{~Hz}$ and $1230 \mathrm{~Hz}$ for neutron stars described by nuclear EOS (see also [29, 4]). The 3rd PN approximations for point masses derived by different authors are giving ISCO at very high frequencies of gravitational waves $>2 \mathrm{kHz}([7,12,13])$.

It should be mentioned that the frequency at ISCO found in quasi-equilibrium approximation could differ from that obtained by the numerical integration of the full set of time dependent Einstein equations. The only comparison (according to the authors' knowledge) between these two approches for binary neutron stars can be found in Marronetti et al. (2004) who showed that for a polytropic EOS with $\gamma=2$ and $M / R=0.14$ the frequency at the ISCO at quasi-equilibrium approximation is $\sim 15 \%$ higher than the dynamical ISCO. Thus full relativistic hydrodynamical calculations are required in order to obtain detailed predictions for particular forms of the nuclear matter equations of state.

\section{Acknowledgements}

This work was partially supported by the "Ayudas para movilidad de Profesores de Universidad e Invesigadores espanoles y extranjeros" program of the Spanish MEC, by the grants 1 P03D 005 30, PBZ-KBN-054/P03/2001 and 5 P03D 01721, by the "Bourses de recherche 2004 de la Ville de Paris" and by the Associated European Laboratory Astro-PF (Astrophysics PolandFrance). DGR is grateful to the COSPAR meeting organizers for support. MB was partially supported by the Marie Curie Intra-european Fellowship MEIF-CT-2005-023644. 


\section{References}

[1] C. Alcock, E. Farhi, and A. Olinto, Astrophys. J. 310, 261 (1986).

[2] A. Akmal , V. R. Pandharipande and D. G. Ravenhall, Phys. Rev. C 58, 1804 (1998)

[3] T. W. Baumgarte and S. L. Shapiro, Phys. Rep. 376, 41 (2003)

[4] M. Bejger, Gondek-Rosinska, Gourrgoulhon, et al., A\&A, 431, 297 (2005)

[5] K. Belczynski, V. Kalogera, and T. Bulik Astrophys. J. 572, 407 (2002).

[6] L. Bildsten and C. Cutler, Astrophys. J. 400, 175 (1992).

[7] L. Blanchet, Phys. Rev. D 65, 124009 (2002).

[8] I. Bombaci in Perspectives on Theoretical Nuclear Physics, 223 (1995)

[9] T. Bulik, D. Gondek-Rosińska, K. Belczynski, MNRAS 352, 1372 (2004).

[10] M. Burgay et al., Nature, 426, 531 (2003).

[11] M. Burgay, N. D'Amico, A. Possenti et al. The Highly Relativistic Binary Pulsar PSR J0737-3039A: Discovery and Implications, astro-ph/0405194.

[12] T. Damour, P. Jaranowski, and G. Schäfer, Phys. Rev. D 62, 044024 (2000).

[13] T. Damour, E. Gourgoulhon, and P. Grandclément, Phys. Rev. D 66, 024007 (2002)

[14] J. A. Faber et al., Phys. Rev. Let. 89, 231102 (2002)

[15] E. Fahri, and R. L. Jaffe, Phys. Rev. D 30, 112379 (1984)

[16] J. L. Friedman, K. Uryu, and M. Shibata, Phys. Rev. D 65, 064035 (2002).

[17] E. Gourgoulhon et al., Phys. Rev. D 63, 064029 (2001)

[18] D. Gondek-Rosińska, E. Gourgoulhon and P. Haensel, A \& A 412, 777 (2003).

[19] D. Gondek-Rosińska, T. Bulik, K. Belczynski, Memorie della Societa Astronomica Italiana, v.76, 513 (2005)

[20] N. K. Glendenning ApJ 293, 470 (1985)

[21] P. Haensel, J.L. Zdunik, and R. Schaeffer, A \& A 160, 121 (1986).

[22] S. A. Hughes, Phys. Rev. D 66, 102001 (2002).

[23] V. Kalogera et al. ApJ 601, 179 (2004).

[24] C. S. Kochanek, Astrophys. J. 398, 234 (1992).

[25] F. Limousin, D. Gondek-Rosińska and E. Gourgoulhon, Phys. Rev. D, $71,064012(2005)$

[26] D. R. Lorimer, Living Rev. Relativity 4, 5 (2001)

[27] J. Madsen, Lect. Notes Phys. 516, 162 (1999).

[28] P. Marronetti et al., Phys. Rev. Lett. 92, 141101 (2004).

[29] R. Oechslin et al., MNRAS 349, 1469 (2004)

[30] K. Taniguchi, E. Gourgoulhon, and S. Bonazzola, Phys. Rev. D 64, 064012 (2001)

[31] K. Taniguchi and E. Gourgoulhon, Phys. Rev. D 66, 104019 (2002)

[32] K. Taniguchi and E. Gourgoulhon, Phys. Rev. D 68, 124025 (2003)

[33] F. Weber, Prog. Part. Nucl. Phys. 54, 193 (2005) 